\title{
Low-speed rotational atherectomy with substantial debulking and long-term survival: a retrospective observational study of 889 consecutive patients
}

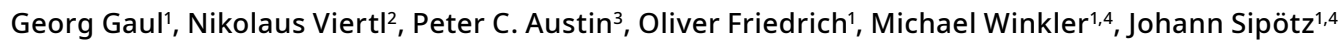 \\ 1 Karl Landsteiner Institute for Scientific Research in Clinical Cardiology, Vienna, Austria \\ 2 Austrian Institute of Technology, Vienna, Austria \\ 3 Institute for Clinical Evaluative Sciences, Toronto, Canada \\ 4 Department of Cardiology, Hanusch-Krankenhaus, Vienna, Austria
}

\section{KEY WORDS}

coronary artery

disease, rotational atherectomy
Correspondence to: Oliver Friedrich PhD, Hanusch Krankenhaus, II. Medical Department Cardiology, Karl Landsteiner Institute for Scientific Research in Clinical Cardiology, Heinrich-Collin-Str-30, A-1140 Vienna, Austria, phone: $+43(0)$ 680-2123203, email: o.friedrich@karl-landsteiner.at Received: January 21, 2019. Revision accepted: February 25, 2019. Published online: February 25, 2019. Kardiol Pol. 2019; 77 (4): 451-457 doi:10.5603/KP.a2019.0035 Copyright by Polskie Towarzystwo Kardiologiczne, Warszawa 2019

\section{ABSTRACT}

BACKGROUND In recent years, a series of studies have investigated long-term outcome of rotational atherectomy (RA). However, only little attention was given to the procedural details of RA technique, especially with regard to platform speed.

AIMS The aim of the study was to evaluate the impact of low-speed RA with substantial debulking before stenting on long-term mortality.

METHODS A group of 356 patients with a high plaque burden underwent substantial debulking with RA with low platform speed of $135000 \mathrm{rpm}$ and was compared with a group of 553 patients treated without $\mathrm{RA}$ in the same time period. In both cohorts lesion preparation was followed by stent implantation. The endpoint was all-cause mortality up to 80 months with a mean (SD) follow-up of 49 (24) months.

RESULTS Despite the fact that patients treated with RA were significantly older and presented more unfavorable lesion characteristics than non-RA patients, there was no significant difference in long-term survival between groups. A propensity analysis with 279 matched pairs showed that long-time survival in RA patients was better than in non-RA patients (hazard ratio, $0.52 ; \mathrm{CI}, 0.32-0.85 ; P<0.01$ ).

CONCLUSIONS Substantial debulking with RA continues to play a role in the treatment of calcified coronary arteries. We hypothesize that RA with low platform speed and substantial debulking with a burr-to-artery ratio of up to 0.64 results in favorable long-time survival in patients with high plaque burden. However, this can only be proved in a prospective randomized trial.

INTRODUCTION For more than 30 years, different debulking methods have been used to minimize arterial recoil after lesion dilation, including directional atherectomy with large blade cutters, ${ }^{1}$ laser angioplasty, or rotational atherectomy (RA). However, none of them has shown long-term benefits. ${ }^{2}$ Rotational atherectomy, first described in the late 1980s as a debulking method for peripheral lesions ${ }^{3}$ and subsequently for coronary disease, ${ }^{4}$ also failed to prove superiority over simple balloon dilation, $, 5,6$ before stenting, ${ }^{7}$ or in the treatment of in-stent restenosis ${ }^{8}$ within a 1-year follow-up. Today, the use of the Rotablator (Boston Scientific Scimed, Maple Grove, Minnesota) is predominantly limited to strongly calcified lesions, uncrossable or undilatable lesions as bailout option, and bifurcated lesions to avoid plaque shift.

There have been only few studies focusing on procedural details of RA. The STRATAS (Study to Determine Rotablator and Transluminal Angioplasty Strategy) ${ }^{9}$ examined the amount of 


\section{WHAT'S NEW?}

Our retrospective single-center study indicates that rotational atherectomy (RA) with low platform speed and substantial debulking with burr-to-artery ratio greater than 0.6 may result in favorable long-term survival in patients with a high plaque burden. We found that patients with RA, who were older and presented significantly more complex lesions, had a similar long-term survival compared with less sick patients without RA. After compensating for differences in baseline variables including age and variables describing lesion morphology with propensity score matching, the long-term survival was even better in RA patients. Prospective randomized trials are needed to confirm the results of our exploratory hypothesis-generating analysis.

debulking with different burr-to-artery (b/a) ratios, with the subsequent recommendation of a $\mathrm{b} / \mathrm{a}$ ratio of less than 0.7 . Questions relating to the speed of the burr and its effects have been systematically investigated only in bench tests. A series of studies have demonstrated that high burr speeds of more than $140000 \mathrm{rpm}$ cause significantly more microcavitations, ${ }^{10}$ activation of platelets, and hemolysis. ${ }^{11-13}$ Two studies in the late 1990s and early 2000s reported favorable outcome by using a platform speed of less than $160000 \mathrm{rpm} .{ }^{14,15}$ In the light of these studies, we modified the technique in our laboratory with short runs, intermittent ablation, and minimal deceleration - the pecking technique-aiming for substantial debulking with a b/a ratio between 0.6 and 0.7 and a platform speed of $135000 \mathrm{rpm}$.

This retrospective observational analysis compares the outcome of patients with a single-vessel intervention treated with this RA strategy with patients treated with conventional ballooning. In both groups lesion preparation was followed by stent implantation.

METHODS Study population During a 6-year period, 1716 consecutive patients were selected for analysis. A total of 1033 patients underwent single-vessel intervention, defined as the only interventional treatment within 8 weeks. All clinical variables were taken from clinical records and entered into a database; angiographic variables were measured by trained personnel not involved in the procedure. As many adverse outcome measurements in a long-term retrospective analysis may be unreliable (eg, myocardial infarction, hospital readmission, stent restenosis), we decided to restrict our long-term outcome parameter to the only undisputed endpoint, death. By checking patient data in the central database of the Austrian social security system, we could define the vital status of every patient ensuring complete data collection. Permission for this reporting was approved by the local ethics committee.

Procedure Rotational atherectomy The RA procedures began with the smallest meaningful burr size, and the step-burr technique was applied. Guiding catheters up to 8F in diameter were used. Three of the authors (JS, MW, GG) were operators with equal experience. We modified the recommendation of the standard protocol by reducing the platform speed outside the patient to $135000 \mathrm{rpm}$. We used a standard solution of verapamil, nitroglycerine, and heparin as rinsing fluid for the inner lumen of the Rotablator. We rarely needed a pacemaker to counteract sinus bradycardia or sinus standstill; instead we administered atropine boluses and, when needed, theophylline infusions (0.48 $\mathrm{mg}$ theophylline in $250 \mathrm{ml}$ saline).

Indications for rotational atherectomy The common indication for RA was the presence of a severe sclerotic, calcified lesion burden. Atherectomy was used in ostial lesion to minimize plaque shifting. Stenting after debulking or predilation followed the usual recommendations for lesion preparation and stenting. ${ }^{16}$ In the cases of uncertain vessel size, intravascular ultrasound was used at the discretion of the interventionalist. Rotational atherectomy was not used in lesions without heavy lesion burden or in less tight lesions. The stents were always implanted with high-pressure inflation up to 20 bars; in the cases of failed expansion, postdilation with a noncompliant balloon was applied.

Angiographic evaluation For quantitative coronary angiography, we used the QUANTCORQCA (CAAS II) 5.0 software (Pie Medical Imaging, Maastricht, the Netherlands). Measurements were made on end-diastolic frames. Reference vessel diameter, percent diameter stenosis, lesion length, and minimal luminal diameter measurements were made before and immediately after stenting. The American College of Cardiology and the American Heart Association classification system ${ }^{17}$ was used for morphological classification of lesions.

Follow-up Patients were typically discharged between day 1 and day 3 after the procedure. After discharge, for logistic reasons clinical followup was not possible for all patients. Therefore, to get valid information about the relevance of our treatment, long-term survival was tracked by the Austrian social security system.

Statistical analysis Patients were analyzed according to their plaque treatment before stenting: those with and without RA. We summarized baseline characteristics of the entire sample with mean values and SDs for continuous variables, and percentages for discrete variables. Differences in baseline characteristics between groups of patients were evaluated using the $t$ test or Wilcoxon rank sum test for continuous variables, and the $\mathrm{X}^{2}$ test or Fisher exact test for categorical variables, as appropriate. 


\begin{tabular}{|c|c|c|c|c|c|c|c|}
\hline \multirow[t]{2}{*}{ Parameter } & \multicolumn{4}{|c|}{ Entire sample } & \multicolumn{3}{|c|}{ Matched pairs } \\
\hline & $\begin{array}{l}\text { Non-RA } \\
\quad(n=533)\end{array}$ & $\begin{array}{l}\text { RA } \\
\quad(n=356)\end{array}$ & SMD & $P$ value & $\begin{array}{l}\text { Non-RA } \\
\quad(n=279)\end{array}$ & $\begin{array}{l}\text { RA } \\
\qquad(n=279)\end{array}$ & SMD \\
\hline Age, y, mean (SD) & $65.9(11.2)$ & $68.5(10.3)$ & 0.26 & 0.001 & $67.5(10.5)$ & $67.9(10.7)$ & 0.04 \\
\hline Male sex, \% & 69 & 69 & 0.00 & NS & 69 & 69 & 0.01 \\
\hline BMI, kg/m², mean (SD) & $29.5(5.6)$ & $28.7(4.5)$ & 0.17 & NS & $28.7(5.3)$ & $28.9(4.5)$ & 0.05 \\
\hline $\mathrm{DM}, \%$ & 35 & 38 & 0.06 & NS & 36 & 37 & 0.02 \\
\hline IDDM, \% & 3 & 4 & 0.06 & NS & 3 & 3 & 0.02 \\
\hline Hypercholesterolemia, \% & 81 & 77 & 0.09 & NS & 80 & 79 & 0.01 \\
\hline Hypertension & 81 & 85 & 0.09 & NS & 84 & 84 & 0.00 \\
\hline Previous MI, \% & 35 & 36 & 0.01 & NS & 35 & 34 & 0.01 \\
\hline Previous CABG, \% & 9 & 12 & 0.08 & NS & 11 & 10 & 0.01 \\
\hline Previous PCI, \% & 12 & 16 & 0.11 & NS & 13 & 14 & 0.02 \\
\hline
\end{tabular}

$A P$ value of less than 0.05 was considered significant.

Abbreviations: BMI, body mass index; CABG, coronary artery bypass grafting; DM, diabetes mellitus; IDDM, insulin-dependent diabetes mellitus; MI, myocardial infarction; NS, nonsignificant; PCI, percutaneous coronary intervention; RA, rotational atherectomy; SMD, standardized mean differences

Cumulative incidence rates (survival curves) were estimated by the Kaplan-Meier method and compared by a log-rank test. Hazard ratios (HRs) were estimated by fitting a Cox proportional hazards model. Propensity-score matching was used to reduce treatment-selection bias for RA. ${ }^{18-20}$ The propensity score was estimated using a logistic regression model, in which treatment assignment (RA vs non-RA) was regressed on prognostically important baseline covariates. ${ }^{21}$ All prognostically important and available covariates were included in the propensity score model (listed in TABLES1 and 2). Exposed and unexposed patients were matched on the logit of the propensity score using nearest-neighbor matching without replacement within calipers defined to be $0.1 \mathrm{SD}$ of the logit of the propensity score. ${ }^{22,23}$ We compared the similarity of baseline covariates between treated and untreated patients in the matched sample, using standardized mean differences, that is, the absolute value of the difference in means relative to the average SD. ${ }^{24}$ Outcomes were compared between treated and untreated subjects in the propensity score-matched sample, using methods appropriate for matched-pair data. ${ }^{25}$ We estimated Kaplan-Meier curves for time-to-event outcomes and tested the equality of the estimated survival curves using the stratified logrank test. The HR for the effect of RA on the hazard of mortality was estimated using a Cox proportional hazards model that stratified the matched pairs to account for the matched nature of the propensity score-matched sample. Continuous and dichotomous outcomes were compared between groups in the matched sample using paired $t$ test and McNemar test respectively. ${ }^{26}$ All $P$ values were 2 -tailed, and a $P$ value of less than 0.05 was considered significant. All CIs were stated at $95 \%$ confidence level. All analyses were performed using the R software, version R 2.11.1 (The R Foundation for Statistical Computing, Vienna, Austria) ${ }^{27}$ on a Mac OSX 10.6 platform (Apple Inc., Cupertino, California, United States) with Intel Core 2 Duo central processing unit. The R package "MatchIt" was used for the propensity score matching.

RESULTS We assessed 1033 consecutive patients treated with a single-vessel intervention. Due to incomplete data, we excluded 144 patients from the analysis and the final sample size comprised 889 patients, 356 of whom (40\%) underwent an interventional procedure with RA. As we are aware of the shortcomings of a retrospective analysis, we analyzed baseline data of all patients without treatment versus those treated with RA with comparable data from the propensity analysis (TABLES 1-3).

Baseline data Patients with RA were significantly older, but did not differ with regard to other clinical variables (TABLE 1).

We showed differences between groups in baseline angiographic data (TABLE2). Patients with RA had more vessels diseased, lesions were longer and more severe, and they had significantly more type $C$ lesions. Rotational atherectomy was used more often in anterior descending and 
TABLE 2 Baseline angiographic parameters before intervention in patients who underwent rotational atherectomy (RA) and those who did not (non-RA)

\begin{tabular}{|c|c|c|c|c|c|c|c|c|}
\hline \multirow[t]{2}{*}{ Parameter } & & \multicolumn{4}{|c|}{ Entire sample } & \multicolumn{3}{|c|}{ Matched pairs } \\
\hline & & $\begin{array}{l}\text { Non-RA } \\
(n=533)\end{array}$ & $\begin{array}{l}\text { RA } \\
(n=356)\end{array}$ & SMD & $P$ value & $\begin{array}{l}\text { Non-RA } \\
(n=279)\end{array}$ & $\begin{array}{l}\text { RA } \\
(n=279)\end{array}$ & SMD \\
\hline \multirow{3}{*}{$\begin{array}{l}\text { Number of } \\
\text { affected } \\
\text { vessels, \% }\end{array}$} & 1 & 44 & 29 & 0.31 & \multirow[t]{3}{*}{$<0.001$} & 35 & 33 & 0.04 \\
\hline & 2 & 27 & 30 & 0.06 & & 25 & 30 & 0.12 \\
\hline & 3 & 29 & 41 & 0.24 & & 40 & 37 & 0.07 \\
\hline \multirow{5}{*}{$\begin{array}{l}\text { Vessel treated, } \\
\%\end{array}$} & LM & 0 & 1 & 0.05 & \multirow[t]{5}{*}{$<0.008$} & 0 & 1 & 0.10 \\
\hline & LAD & 42 & 50 & 0.18 & & 44 & 48 & 0.09 \\
\hline & $C x$ & 23 & 15 & 0.18 & & 17 & 18 & 0.04 \\
\hline & $\mathrm{RCA}$ & 32 & 33 & 0.01 & & 38 & 32 & 0.13 \\
\hline & SVG & 3 & 1 & 0.28 & & 1 & 1 & 0.04 \\
\hline \multirow{3}{*}{$\begin{array}{l}\text { Type of lesion } \\
\text { (ACC/AHA), \% }\end{array}$} & A & 18 & 5 & 0.40 & \multirow[t]{3}{*}{$<0.001$} & 5 & 7 & 0.08 \\
\hline & $\mathrm{B}$ & 47 & 33 & 0.29 & & 40 & 38 & 0.03 \\
\hline & C & 35 & 62 & 0.54 & & 55 & 55 & 0.01 \\
\hline \multirow[t]{7}{*}{ Other variables } & RVD, mm, mean (SD) & $3.0(0.9)$ & $2.9(0.6)$ & 0.19 & NS & $3.0(0.9)$ & $2.9(0.6)$ & 0.09 \\
\hline & MLD, mm, mean (SD) & $0.9(0.5)$ & $0.8(0.4)$ & 0.25 & $<0.001$ & $0.8(0.4)$ & $0.8(0.4)$ & 0.02 \\
\hline & $\begin{array}{l}\text { Lesion length, mm, mean } \\
\text { (SD) }\end{array}$ & $10.0(7.1)$ & $13.5(10.6)$ & 0.28 & $<0.001$ & $11.6(8.1)$ & $12.3(9.1)$ & 0.06 \\
\hline & Stenosis, \%, mean (SD) & $68.6(15.3)$ & $71.1(13.8)$ & 0.18 & $<0.007$ & $71.0(15.4)$ & $70.3(14.3)$ & 0.05 \\
\hline & Restenosis, \% & 2.3 & 4.2 & 0.08 & NS & 2 & 2 & 0.02 \\
\hline & Ostial stenosis, \% & 7.2 & 17.4 & 0.27 & $<0.001$ & - & - & - \\
\hline & Bifurcation, $\%$ & 6 & 9 & 0.11 & NS & - & - & - \\
\hline
\end{tabular}

A $P$ value of less than 0.05 was considered significant.

Abbreviations: ACC/AHA, American College of Cardiology and American Heart Association; $C x$, circumflex artery; LAD, left anterior descending coronary artery; LM, left main coronary artery; MLD, minimal lumen diameter; RCA, right coronary artery; RVD, reference vessel diameter; SVG, saphenous vein graft; others, see TABLE 1

TABLE 3 Interventional parameters of patients who underwent rotational atherectomy (RA) and those who did not (non-RA)

\begin{tabular}{lllllll} 
Parameter & \multicolumn{3}{c}{ Entire sample } & \multicolumn{3}{c}{ Matched pairs } \\
& $\begin{array}{c}\text { Non-RA } \\
(\mathrm{n}=533)\end{array}$ & $\begin{array}{c}\text { RA } \\
(\mathrm{n}=356)\end{array}$ & $\begin{array}{c}\text { Pvalue } \\
\text { Final lumen, mm, mean (SD) }\end{array}$ & $\begin{array}{c}\text { Non-RA } \\
(\mathrm{n}=279)\end{array}$ & $\begin{array}{c}\text { RA } \\
(\mathrm{n}=279)\end{array}$ & Pvalue \\
\hline Lumen gain, mm, mean (SD) & $2.19(0.6)$ & $2.1(0.5)$ & NS & $3.1(0.5)$ & $3.2(0.5)$ & NS \\
\hline Stent length, mm, mean (SD) & $15.9(7.3)$ & $18.2(9.5)$ & NS & $17.1(8.0)$ & $17.8(9.4)$ & NS \\
\hline Number of stents, mean (SD) & $1.4(0.8)$ & $1.6(0.8)$ & NS & $1.5(0.8)$ & $1.6(0.8)$ & NS \\
\hline DES, \% & 28.3 & 23.1 & NS & 18 & 22 & NS \\
\hline IVUS, \% & 13.3 & 20.2 & $<0.007$ & 15.7 & 19.7 & NS \\
\hline Max burr length, mm, mean (SD) & - & $1.8(0.3)$ & - & - & $1.8(0.3)$ & - \\
\hline Burr-to-artery ratio, mean (SD) & - & $0.64(0.14)$ & - & - & $0.6(0.1)$ & - \\
\hline
\end{tabular}

A $P$ value of less than 0.05 was considered significant.

Abbreviations: DES, drug-eluting stent; IVUS, intravascular ultrasound; others, see TABLES 1 and 2 

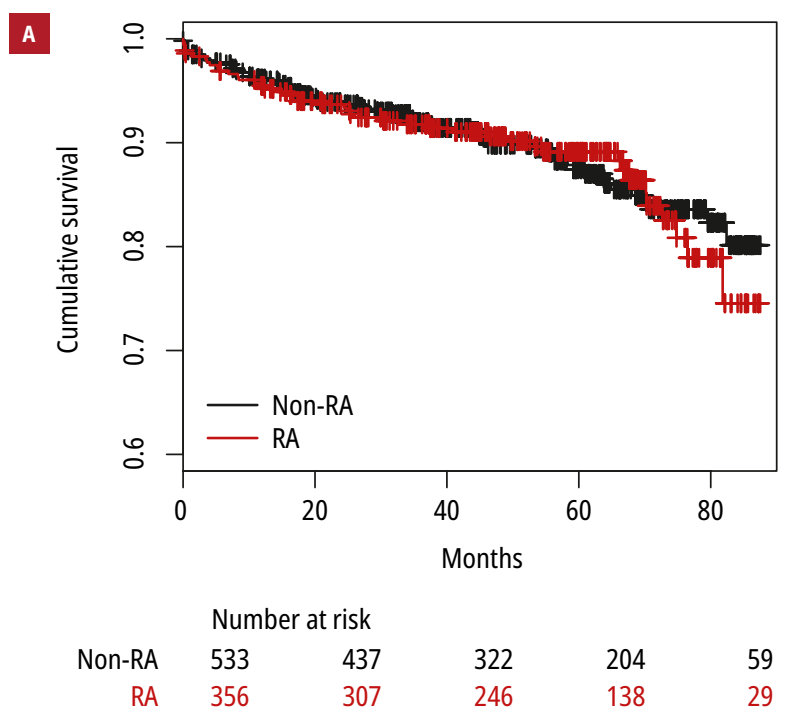
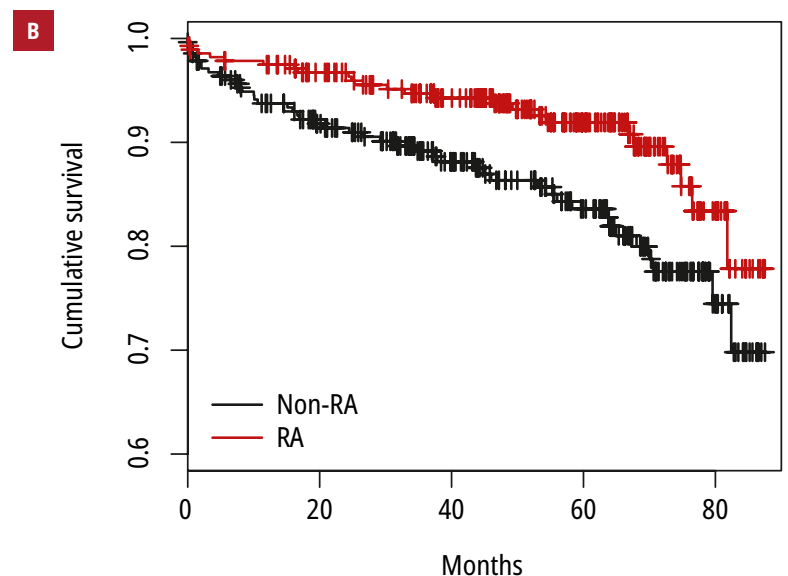

\begin{tabular}{rlrrrr}
\multicolumn{7}{l}{ Number at risk } \\
Non-RA & 279 & 226 & 165 & 112 & 22 \\
RA & 279 & 250 & 208 & 113 & 26
\end{tabular}

FIGURE 1 The Kaplan-Meier overall survival analysis of all patients who underwent rotational atherectomy (RA) and those who did not (non-RA) (A) and matched pairs (B) up to 80 months

less often in circumflex lesions, and it was applied more often in the treatment of ostial lesions in an attempt to reduce the shift of the carina and to exert less barotrauma in these delicate lesions.

Procedural variables after intervention The analysis of the entire sample resulted in a similar final lumen diameter in both groups, and the baseline minimal lumen diameter was smaller in the RA group. Therefore, lumen gain after stenting was significantly greater in the RA group. The length of stented segments, as well as the number of stents and drug-eluting stents (DESs) were the same in both groups. The use of intravascular ultrasound was significantly higher in the RA group because of the complexity of the disease. The mean b/a ratio was 0.64 (TABLE3).

Kaplan-Meier analysis of long-term overall survival Comparing all RA and nonRA patients, we found a complete overlap of Kaplan-Meier survival curves (HR, 1.05; 95\% CI, 0.71-1.55; $P=0.82$ ) (FiguRE 1A). Propensity score matching resulted in 279 matched pairs (ie, 558 patients) out of 889 patients, which means that $78.4 \%$ of the RA-treated patients could successfully be matched to an RA-untreated patient. Prior to matching, the maximum standardized difference across the 26 baseline variables was 0.41 . This decreased to 0.08 after matching (TABLES1 and 2). A threshold of 0.1 is commonly used to indicate acceptable balance. ${ }^{26}$ Kaplan-Meier survival plots of matched pairs demonstrated a significant advantage for the RA group (HR, 0.52; 95\% CI, 0.32-0.85; $P<0.01$ ) (Figure 1B).

DISCUSSION In our study, we compared 356 patients after RA with 533 patients after conventional lesion preparation. Despite significantly worse baseline conditions (RA patients were significantly older and presented a significantly more unfavorable lesion morphology), we found a striking complete overlay of Kaplan-Meier curves for overall long-term mortality. This suggests that vessel preparation with RA allows to increase survival of patients with complex calcified lesions to the level of patients with less severe coronary disease treated with standard percutaneous coronary intervention (PCI) without prior debulking by RA. To confirm our finding, we performed a propensity score matching procedure compensating for differences between the samples including age and variables describing lesion morphology. When 279 matched pairs were compared, a better overall survival in patients treated with RA and substantial debulking was found.

Only 2 recent studies directly compared long-time mortality between RA and non-RA patients. Cockburn et al ${ }^{28}$ reported the outcomes of PCI patients in the United Kingdom over a 3.5year follow-up, based on data from the United Kingdom Central Cardiac Audit Database. The comparison of 2154 patients treated with RA with a large cohort of non-RA patients revealed a higher overall mortality in patients treated with RA (HR, 2.1). After compensating for differences in baseline clinical variables with propensity score matching, the difference between the 2 groups shrunk considerably (HR, 1.26) but remained significant. Armstrong et a ${ }^{29}$ reported no difference in a propensity-weighted comparison of 2-year mortality between RA and non-RA patients in a study including only patients with calcified lesions. These results seem to disagree with our findings. However, 2 points have to be considered in this context. First and foremost, both analyses comprised the results of a variety 
of centers with presumably varying RA protocols and differing institutional volumes of RA cases. Our study included patients from 1 center and had a single, consistent RA protocol carried out by a small group of interventionists of equal and considerable experience. This is especially important since a recent study analyzing data of 13335 RA patients of the Japanese nationwide PCI registry showed that high institutional volume is associated with favorable outcome of RA, ${ }^{30}$ which has led to the recommendation that RA should only be performed in selected facilities with personnel highly familiar with the complex RA procedure. ${ }^{31}$ Second, in contrast to our analysis, the propensity score matching procedure used to adjust for baseline clinical characteristics in these studies did not take account of variables describing important aspects of vessel morphology such as the length of stenosis or minimal lumen diameter.

All studies dealing with RA should also be discussed in regard to the technique of atherectomy. Different technical protocols of RA have been addressed in only very few clinical reports. In STRATAS, ${ }^{9}$ the relation of the burr size to the reference vessel lumen was analyzed and resulted in the final recommendation of a b/a ratio of less than 0.7 , followed by a balloon dilation with a pressure of less than 4 bars. The burr speed has never been investigated in a randomized trial. Uetani et $\mathrm{a}^{14}$ compared 2 different burr speed protocols and identified low platform speed as an independent predictor of favorable outcome: with a burr speed of 150000 to $160000 \mathrm{rpm}$, they found a 6 -month binary restenosis rate of $33.8 \%$, whereas a protocol with a speed of 170000 to $190000 \mathrm{rpm}$ resulted in a higher binary restenosis rate of $57.9 \%$. Kiesz et $\mathrm{al}^{15}$ achieved a low binary restenosis rate of $28.1 \%$ with balloon dilation in patients with very long calcified lesions only after changing the RA protocol to a burr speed of 140000 to $160000 \mathrm{rpm}$ and an intended b/a ratio of 0.75 . In a recent study, Mezilis et $\mathrm{al}^{32}$ reported favorable results with the combination of RA at a speed below $140000 \mathrm{rpm}$ plus the use of DES. However, despite these positive results and bench tests of Reisman et $\mathrm{al}^{11,13}$ in the late 1990s indicating a potential benefit of lower platform speed, most trials were conducted with burr speeds higher than $160000 \mathrm{rpm}$. In the latest randomized study ROTAXUS (Rotational Atherectomy Prior to Taxus Stent Treatment for Complex Native Coronary Artery Disease), Abdel-Wahab et $\mathrm{al}^{33}$ used a technique with an average speed of $167000 \mathrm{rpm}$ and a b/a ratio of 0.48 . They found that the significantly higher procedural lumen gain in the RA group was counterbalanced by increased late lumen loss and concluded that vessel preparation with RA does not improve efficiency of DES.

Our results for RA were achieved with a low burr speed (135 rpm) and substantial debulking (b/a ratio, 0.64). The latest recommendations on RA discuss a burr speed between $135000 \mathrm{rpm}$ and $180000 \mathrm{rpm}$, whereas a b/a ratio of 0.6 should be attained. ${ }^{34}$ We hypothesize that our protocol combining low burr speed and substantial debulking may be responsible for the favorable outcome in long-term survival. However, this can be ascertained only in a randomized trial.

Limitations The study has several limitations. First, this is a retrospective study with all the inherent limitations of a nonprospective study design. Second, the indication for rotablation was defined by the plaque burden; however, there was no clear-cut recommendation for the use of a rotablator. Although we performed a single-center study with a few interventionalists of equal experience, which usually guarantees a consistent use of the recommended technique, even the most experienced interventionalists have their own approach to a given intervention.

Conclusions This is a retrospective singlecenter investigation on preparation of severe lesions before stenting, using a protocol of RA with a low platform speed and a b/a ratio greater than 0.6, resulting in substantial debulking. It was demonstrated that older patients with more severe lesions treated with RA achieved a similar long-term survival as younger patients with a smaller number of diseases after lesion preparation with conventional ballooning. After adjusting for differences between the groups with propensity score matching, we found a significantly better long-term survival in patients after RA. Therefore, it could be speculated that patients with complex lesions with high plaque burden in the non-RA group could potentially benefit from RA. However, this result can only be verified by a prospective randomized trial as our study may be understood as hypothesis generating. Further research with special focus on the procedural details of RA is needed to explore the potential benefit of RA in patients with a high plaque burden.

\section{ARTICLE INFORMATION}

ACKNOWLEDGMENTS The study was not supported by any grants.

CONFLICT OF INTEREST JS and GG are proctors for RA and received teaching fees from Boston Scientific. They declare no other conflict of interest in regard to the study. The other authors declare no conflict of interest in regard to the study. HOW TO CITE Gaul G, Viertl N, Austin PC, et al. Low-speed rotational atherectomy with substantial debulking results in favorable long-term survival: a retrospective observational study of 889 consecutive cases. Kardiol Pol. 2019; 77: 451-457. doi:10.5603/KP.a2019.0035

\section{REFERENCES}

1 Baim DS, Cutlip DE, Sharma SK, et al. Final results of the balloon vs optimal atherectomy trial (BOAT). Circulation. 1998; 97: 322-331.

2 Reifart N, Vandormael M, Krajcar M, et al. Randomized comparison of angioplasty of complex coronary lesions at a single center. Circulation. 1997; 96: 91-98.

3 Zacca NM, Raizner AE, Noon GP, et al. Treatment of symptomatic peripheral atherosclerotic disease with a rotational atherectomy device. Am J Cardiol. 1989; 63: 77-80. 
4 Erbel R, O'Neill W, Auth D, et al. High-frequency rotational atherectomy in coronary heart disease [in German]. Dtsch Med Wochenschr. 1989; 114: 487-495.

5 Dill T. A randomized comparison of balloon angioplasty versus rotational atherectomy in complex coronary lesions (COBRA study). Eur Heart J. 2000; 21: 1759-1766.

6 Mauri L, Reisman M, Buchbinder M, et al. Comparison of rotational atherectomy with conventional balloon angioplasty in the prevention of restenosis of small coronary arteries. Am Heart J. 2003; 145: 847-854

7 Cavusoglu E, Kini AS, Marmur JD, Sharma SK. Current status of rotational atherectomy. Catheter Cardiovasc Interv. 2004; 62: 485-498.

8 vom Dahl J. Rotational atherectomy does not reduce recurrent in-stent restenosis: results of the angioplasty versus rotational atherectomy for treatment of diffuse in-stent restenosis trial (ARTIST). Circulation. 2002; 105: 583-588.

9 Whitlow PL, Bass TA, Kipperman RM, et al. Results of the study to determine rotablator and transluminal angioplasty strategy (STRATAS). Am J Cardiol. 2001; 87: 699-705.

10 Zotz RJ, Erbel R, Philipp A, et al. High-speed rotational angioplasty-induced echo contrast in vivo and in vitro optical analysis. Cathet Cardiovasc Diagn. 1992; 26: 98-109.

11 Reisman M, Shuman BJ, Dillard D, et al. Analysis of low-speed rotational atherectomy for the reduction of platelet aggregation. Cathet Cardiovasc Diagn. 1998; 45: 208-214.

12 Williams MS, Coller BS, Väänänen HJ, et al. Activation of platelets in plateletrich plasma by rotablation is speed-dependent and can be inhibited by abciximab (c7E3 Fab; ReoPro). Circulation. 1998; 98: 742-748.

13 Reisman M, Shuman BJ, Harms V. Analysis of heat generation during rotational atherectomy using different operational techniques. Cathet Cardiovasc Diagn. 1998; 44: 453-455.

14 Uetani T, Ishii H, Sakai S, et al. Beneficial effect of rotational atherectomy with low platform speed on late outcomes. Int J Cardiol. 2004; 94: 35-40.

15 Kiesz RS, Rozek MM, Ebersole DG, et al. Novel approach to rotational atherec tomy results in low restenosis rates in long, calcified lesions: long-term results of the San Antonio rotablator study (SARS). Catheter Cardiovasc Interv. 1999; 48: 48-53.

16 Silber S, Albertsson P, Avilés FF, et al. Guidelines for percutaneous coronary interventions. Eur Heart J. 2005; 26: 804-847.

17 Ryan TJ, Faxon DP, Gunnar RM, et al. Guidelines for percutaneous transluminal coronary angioplasty. A report of the American College of Cardiology/American Heart Association task force on assessment of diagnostic and therapeutic cardiovascular procedures (subcommittee on percutaneous transluminal coronary angioplasty). Circulation. 1988; 78: 486-502.

18 Rosenbaum PR, Rubin DB. The central role of the propensity score in observational studies for causal effects. Biometrika. 1983; 70: 41.

19 Austin PC. Primer on statistical interpretation or methods report card on propensity-score matching in the cardiology literature from 2004 to 2006. Circ Cardiovasc Qual Outcomes. 2008; 1: 62-67.

20 Austin PC. An introduction to propensity score methods for reducing the ef fects of confounding in observational studies. Multivariate Behav Res. 2011; 46: 399-424.

21 Austin PC, Grootendorst P, Anderson GM. A comparison of the ability of different propensity score models to balance measured variables between treated and untreated subjects: a Monte Carlo study. Stat Med. 2007; 26: 734-753.

22 Austin PC. Balance diagnostics for comparing the distribution of baseline covariates between treatment groups in propensity-score matched samples. Stat Med. 2009; 28: 3083-3107.

23 Austin PC. Optimal caliper widths for propensity-score matching when estimating differences in means and differences in proportions in observational studies. Pharm Stat. 2011; 10: 150-161.

24 Austin PC. A comparison of 12 algorithms for matching on the propensity score. Stat Med. 2014; 33: 1057-1069.

25 Austin PC. Type I error rates, coverage of confidence intervals, and variance estimation in propensity-score matched analyses. Int J Biostat. 2009; 5: Article 13.

26 Normand SL, Morris CN, Fung KS, et al. Development and validation of a claims based index for adjusting for risk of mortality: the case of acute myocardial infarction. J Clin Epidemiol. 1995; 48: 229-243.

27 R Core Team (2014). R: A language and environment for statistical computing. R Foundation for Statistical Computing, Vienna, Austria. http://www.R-project.org/.

28 Cockburn J, Hildick-Smith D, Cotton J, et al. Contemporary clinical outcomes of patients treated with or without rotational coronary atherectomy — an analysis of the UK central cardiac audit database. Int J Cardiol. 2014; 170: 381-387.

29 Armstrong El, Stanislawski MA, Kokkinidis DG, et al. Coronary atherectomy is associated with improved procedural and clinical outcomes among patients with calcified coronary lesions: insights from the VA CART program. Catheter Cardiovasc Interv. 2018; 91: 1009-1017.

30 Sakakura K, Inohara T, Kohsaka S, et al. Incidence and determinants of complications in rotational atherectomy: insights from the national clinical data (J-PC Registry). Circ Cardiovasc Interv. 2016; 9: 1-7.

31 Uetani T, Amano T. Current status of rotational atherectomy in the drug-eluting stent era. Circ J. 2018; 82: 946-947.
32 Mezilis N, Dardas P, Ninios V, Tsikaderis D. Rotablation in the drug eluting era: immediate and long-term results from a single center experience. J Interv Cardiol. 2010; 23: 249-253.

33 Abdel-Wahab M, Richardt G, Joachim Büttner H, et al. High-speed rotational atherectomy before paclitaxel-eluting stent implantation in complex calcified coronary lesions: the randomized ROTAXUS (Rotational Atherectomy Prior to Taxus Stent Treatment for Complex Native Coronary Artery Disease) trial. JACC Cardiovasc Interv. 2013; 6: 10-19.

34 Barbato E, Carrié D, Dardas $P$, et al. European expert consensus on rotational atherectomy. EuroIntervention. 2015; 11: 30-36. 